\title{
1 Analytical models for $\beta$-diversity and the power-law scaling of $\beta$-deviation
}

2

3 Dingliang Xing ${ }^{1,2,3} *$ and Fangliang $\mathrm{He}^{1,2}$

4

51 Department of Renewable Resources, University of Alberta, Edmonton, AB, Canada

62 ECNU-Alberta Joint Lab for Biodiversity Study, Tiantong Forest Ecosystem National

8

Observation and Research Station, School of Ecological and Environmental Sciences, East

China Normal University, Shanghai, China

3 Institute of Eco-Chongming (IEC), Shanghai, China

Email addresses: DX: dlxing@des.ecnu.edu.cn FH: fhe@ualberta.ca

*Correspondence author:

dlxing@des.ecnu.edu.cn

School of Ecological and Environmental Sciences

East China Normal University

500 Dongchuan Road

Shanghai, 200241

China

Authorship: DX and FH conceived the study. DX derived the analytical results and analyzed the data. DX and FH wrote the paper.

Data accessibility: Data and codes supporting the results can be accessed at https://github.com/DLXING/beta analytical .

Running title: analytical models for $\beta$-diversity

Keywords: Beta diversity, log-series distribution, maximum entropy, METE, null model, spatial aggregation, species abundance distribution, species spatial pattern

Words count: abstract: 173; main text: 4603; figure legends: 403

Number of references: 36

Number of figures: 5 


\section{$38 \quad$ ABSTRACT}

39 1. $\beta$-diversity is a primary biodiversity pattern for inferring community assembly. A

40 randomized null model that generates a standardized $\beta$-deviation has been widely used for

41 this purpose. However, the null model has been much debated and its application is limited to

42 abundance data.

43 2. Here we derive analytical models for $\beta$-diversity to address the debate, clarify the

44 interpretation, and extend the application to occurrence data.

453 . The analytical analyses show unambiguously that the standardized $\beta$-deviation is a

46 quantification of the effect size of non-random spatial distribution of species on $\beta$-diversity

47 for a given species abundance distribution. It robustly scales with sampling effort following a

48 power law with exponent of 0.5 . This scaling relationship offers a simple method for

49 comparing $\beta$-diversity of communities of different sizes.

50 4. Assuming logseries distribution for the metacommunity species abundance distribution,

51 our model allows for calculation of the standardized $\beta$-deviation using occurrence data plus a

52 datum on the total abundance.

535 . Our theoretical model justifies and generalizes the use of the $\beta$ null model for inferring

54 community assembly rules.

\section{Keywords}

57 Beta diversity, log-series distribution, maximum entropy, METE, null model, spatial aggregation, species abundance distribution, species spatial pattern 


\section{INTRODUCTION}

61 Comparing $\beta$-diversity, the variation in species composition, among different regions is an

62 important yet controversial topic in ecology (Kraft et al., 2011; Qian, Chen, Mao, \& Ouyang, 2013; Bennett \& Gilbert, 2016; Ulrich et al., 2017). A randomization-based null model that was said to correct for the dependence of raw $\beta$-diversity on species pool (Kraft et al., 2011) has been widely adopted for this purpose (e.g., De Cáceres et al., 2012; Myers et al., 2013; incorrect interpretations of the $\beta$-deviation metric and the dependence of the metric on sampling effort and species pool (Qian et al., 2013; Bennett \& Gilbert, 2016; Ulrich et al., 2017, 2018). Another limitation of the null model is that it requires individual-level data and thus limits its application to situations when only abundances of individual species in each local community are available. Therefore, there is a strong need for a theoretical scrutiny of the null model to justify its use and also a need to extend its application to presence/absence data.

It has been established that the abundance of species and their spatial aggregation in a metacommunity are the two basic quantities that construct macroecological patterns of

77 diversity, including $\beta$-diversity (He \& Legendre, 2002; Plotkin \& Muller-Landau, 2002; Morlon et al., 2008). All ecological processes, biotic and abiotic, are acting through these two quantities to affect the observed macroecological patterns (He \& Legendre, 2002). For instance, both dispersal limitation and environmental filtering can result in spatially aggregated distribution of species, which in turn contributes to high $\beta$-diversity. However, the reverse is not necessarily true: a high $\beta$-diversity does not have to result from aggregated 
distribution. This is because differences in species pool could also alter values of observed

$84 \beta$-diversity. Therefore, it is necessary to disentangle the effect of spatial aggregation from that

of species pool for inferring contributions from different ecological processes on $\beta$-diversity.

distribution. It is thus clear that the $\beta$-deviation is a measure of the effect of non-random

(2011), this interpretation of $\beta$-deviation has not been widely heeded in the literature (e.g.,

Qian et al. 2013). The original interpretation of $\beta$-deviation was "a standard effect size of

$\beta$-deviation measures the effect of species spatial distribution on $\beta$-diversity. 
effort (Bennett \& Gilbert, 2016). In a recent study, we demonstrated by simulation that

$\beta$-deviation can effectively identify and correctly compare non-random $\beta$ patterns across assemblages under the condition of constant sampling effort (see Appendix S2 in Xing \& He 2019). This suggests that there might be some scaling relationship between $\beta$-deviation and the sampling effort. In this study, we show that there is actually a surprisingly simple and robust scaling relationship between $\beta$-deviation and sampling effort. The discovery of this scaling relationship is remarkable. It analytically addresses the criticisms raised by Bennett \& Gilbert (2016) on the sample-effort dependence of $\beta$-deviation.

The last shortcoming of the currently used null model, inherited from the randomization procedure, is that it only deals with abundance data. Yet, in many studies, particularly at regional scales, presence/absence of species are the only data available (e.g., Gaston et al., 2007; McKnight et al., 2007; Buckley \& Jetz, 2008). Therefore, there is a need to extend its application to presence/absence data, so that it could be used in broader contexts such as delineating biogeographical regions (Kreft \& Jetz, 2010).

Here, based on theories about species abundance distribution and spatial distribution of species, we develop an analytical null model to replace the randomization procedure and to address all the above problems. The analytical results reveal that Kraft et al.'s $\beta$-deviation (1) is a measure of the effect size of non-random spatial species distribution on $\beta$-diversity, (2) scales with the sampling effort following a power law with a constant exponent of 0.5 , and (3) can be reasonably estimated based on presence/absence data, provided that a datum on the total abundance of the metacommunity is known (which is usually more readily available than abundance for each species). We test the performance and utility of our analytically derived models using several well-studied empirical data sets. 


\section{DERIVATION OF ANALYTICAL MODELS FOR $\boldsymbol{\beta}$-DIVERSITY}

131

Consider a metacommunity consisting of $M$ equal-sized (equal-area) local communities and there are in total $N$ individuals belonging to $S$ species. The widely used proportional species turnover (denoted $\beta_{\mathrm{P}}$ following Tuomisto (2010)) is defined as $\beta_{\mathrm{P}}=1-\bar{\alpha} / S$, where $\bar{\alpha}$ is the average number of species over the $M$ local communities. Two other major multi-community measures of $\beta$-diversity, namely the $M$-community Sorensen- $\left(\beta_{\mathrm{S}}\right)$ and Jaccard-differentiation $\left(\beta_{\mathrm{J}}\right)\left(\right.$ Chao \& Chiu, 2016), are monotonically related with $\beta_{\mathrm{P}}$ : $\beta_{\mathrm{S}}=\frac{1}{M-1} \frac{\beta_{\mathrm{P}}}{1-\beta_{\mathrm{P}}}, \beta_{\mathrm{J}}=\frac{M}{M-1} \beta_{\mathrm{P}}$. Hence, although we will only focus on $\beta_{\mathrm{P}}$ in this paper, our analytical models can be easily transformed to these other indices.

From the definition and some simple rearrangements (see Appendix S1), it is easy to show that $\beta_{\mathrm{P}}$ is the expectation of the probability that a species from the metacommunity is absent from a local community and thus can be expressed as:

$\beta_{\mathrm{P}}=\sum_{n=1}^{\infty} \Pi(0 \mid n, M) \Phi(n \mid S, N)$,

where $\Pi(0 \mid n, M)$ is the probability that a species with $n$ individuals in the metacommunity is absent from a randomly chosen local community, and $\Phi(n \mid S, N)$ is the metacommunity species abundance distribution (SAD), i.e., the probability of a species having $n$ individuals. This model provides a general framework to develop analytical solutions for Kraft et al.'s null model and the resultant $\beta$-deviation.

\section{Null model: $\beta$-diversity under random spatial distribution of species}

Kraft et al.'s null model randomly shuffles species identities across the $N$ individuals that 
comprise the $M$ local communities while keeping the metacommunity SAD and each of the

local community sizes unchanged. By repeating the randomization procedure a large number

of times (e.g., 999) one can get the expectation $\left(\beta_{\text {null }}\right)$ and corresponding variance $\left(\operatorname{Var}_{\Pi}\left(\beta_{\text {null }}\right)\right)$

of $\beta$-diversity under the null model. Here the subscript $\Pi$ is used to denote that the variation is due to species spatial distribution, in contrast to that due to SAD, which will be made clear

below. This randomization is equivalent to assuming random spatial patterns of species.

$\beta$-deviation is then defined as $\beta_{\text {dev }}=\frac{\beta_{\text {obs }}-\beta_{\text {null }}}{\sqrt{\operatorname{Var}_{\Pi}\left(\beta_{\text {null }}\right)}}$ (Kraft et al. 2011). This standardized metric describes the degree of departure from random distribution in spatial distribution of empirical species, while maintaining the $\operatorname{SAD} \Phi(n \mid S, N)$.

We now use the general formulation of $\beta_{\mathrm{P}}$ as defined by equation (1) to derive the null $\beta$ for random distribution of species in the metacommunity consisting of $M$ local communities.

The probability for a randomly distributed species with abundance $n$ being absent from a local community is: $\Pi(0 \mid n, M)=\left(1-\frac{1}{M}\right)^{n}$. What we need to determine is which SAD should be used in equation (1). The empirical SAD was used in Kraft et al. (2011). It is well established in theory that SAD of metacommunity follows logseries distribution. This is a principal result of the neutral theory of ecology (Hubbell, 2001; Volkov, Banavar, Hubbell, \& Maritan, 2003) and is also predicted by the maximum entropy theory of ecology (Pueyo, He, \& Zillio, 2007; Harte, 2011). In practice, logseries together with lognormal distribution have been repeatedly shown to be the two models fitting empirical data best (McGill et al., 2007) and recent meta-analyses even show logseries outperforms other models (White, Thibault, \& Xiao, 2012; Baldridge, Harris, Xiao, \& White, 2016). In this study, we adopt the theoretical $\log$ series $\mathrm{SAD}$ for metacommunity to derive $\beta_{\text {null }}$ and its variance. We also tested the use of lognormal distribution by simulation but that does not change our main results about the 
173

174

175

176

177

178

179

180

scaling of $\beta$-deviation (see Appendix S2).

For a $\operatorname{logseries} \mathrm{SAD}, \Phi(n \mid S, N)=-\frac{1}{\ln (1-p)} \frac{p^{n}}{n}$, the only parameter $p=e^{-\lambda}$ is fully determined by the state variables $N$ and $S: \frac{N}{S}=\frac{\sum_{n=1}^{N} e^{-\lambda n}}{\sum_{n=1}^{N} e^{-\lambda n} / n}$ (Harte, 2011). Based on this SAD model and the binomial model for random species spatial distribution (Barton \& David, 1959;

He \& Reed, 2006), we derive the following analytical version of the null model (see

Appendix S1 for mathematical details):

$\beta_{\text {null }}=\frac{\ln \left(1-p\left(1-\frac{1}{M}\right)\right)}{\ln (1-p)} \approx \frac{\ln \left(\frac{M}{1+\lambda M}\right)}{\ln (1 / \lambda)}$

$\operatorname{Var}_{\Pi}\left(\beta_{\text {null }}\right)=\frac{1}{S M \ln (1-p)}\left[\ln \left(\frac{M(1-p)+p}{M(1-p)+2 p}\right)\right.$

$$
\begin{aligned}
& \left.-M \ln \left(1-\frac{1}{M(M(1-p) / p+2)}\right)\right] \\
\approx & \frac{1}{S M \ln (1 / \lambda)}\left[\ln \left(\frac{2+\lambda M}{1+\lambda M}\right)-\frac{1}{2+\lambda M}\right] .
\end{aligned}
$$

We note that the approximations are valid under conditions $M \gg 1$ and $\lambda \ll 1$ which are expected for real ecosystems (Harte 2011, p150). This is because in real applications the size of a local community is usually much smaller than the area of the metacommunity $(M=$ metacommunity area / local-community size). However, we would recommend using the exact formulas in real applications, particularly when the sample size is small, since they impose no computing challenge. However, the approximations offer analytical simplicity and we will use them to derive the scaling relationship between $\beta$-deviation and the sampling effort (see below).

From equation (2) it is clear that the expectation and variance of $\beta_{\text {null }}$ under the spatial random distribution are fully determined by the parameter of the logseries $\operatorname{SAD}(p)$, the number of local communities $(M)$, and the total number of species $(S)$. The property that the 
parameter $p$ is fully determined by $S$ and $N$ allows $\beta_{\text {null }}$ to be parameterized using only the

three state variables $N, S$, and $M$. This means we can compare $\beta$-diversity calculated from

occurrence data with the null model as long as the metacommunity size $N$ is known (or can

be estimated). This offers a method of testing $\beta$-diversity in cases where abundances of

individual species are not available. The resultant analytical $\beta$-deviation is obtained by

substituting equation (2) into $\beta_{\mathrm{dev}}=\frac{\beta_{\text {obs }}-\beta_{\text {null }}}{\sqrt{\operatorname{Var}_{\Pi}\left(\beta_{\text {null }}\right)}}$.

The variance in equation (2b) is the expected conditional variance due to species spatial

distribution, i.e., $\operatorname{Var}_{\Pi}\left(\beta_{\text {null }}\right)=\mathrm{E}_{\Phi}\left(\operatorname{Var}_{\Pi}(\beta \mid \Phi)\right)$. No variation due to SAD arises because in

Kraft et al.'s randomization approach the empirical SAD is used. However, in scientific

inference, the empirical SAD ought to be considered as a sample of the underlying theoretical

logseries SAD. It then becomes clear that the sampling procedure introduces additional

variance in the expected $\beta_{\text {null }}$. According to the law of total variance, this second variance can

be written as (Appendix S1): $\operatorname{Var}_{\Phi}\left(\beta_{\text {null }}\right)=\operatorname{Var}_{\Phi}\left(\mathrm{E}_{\Pi}(\beta \mid \Phi)\right)=\frac{1}{S \ln (1-p)}[\ln (1-p(1-$

$\left.\left.\left.\frac{1}{M}\right)^{2}\right)-\frac{1}{\ln (1-p)} \ln ^{2}\left(1-p\left(1-\frac{1}{M}\right)\right)\right]$, where the subscript $\Phi$ indicates that the variation is due to SAD. This leads naturally to a quantification of the variance of $\beta$-deviation due to the

metacommunity SAD:

$$
\begin{aligned}
\operatorname{Var}_{\Phi}\left(\beta_{\mathrm{dev}}\right)= & \frac{\operatorname{Var}_{\Phi}\left(\beta_{\text {null }}\right)}{\operatorname{Var}_{\Pi}\left(\beta_{\text {null }}\right)} \\
= & \frac{M\left[\ln \left(1-p\left(1-\frac{1}{M}\right)^{2}\right)-\frac{1}{\ln (1-p)} \ln ^{2}\left(1-p\left(1-\frac{1}{M}\right)\right)\right]}{\ln \left(\frac{M(1-p)+p}{M(1-p)+2 p}\right)-M \ln \left(1-\frac{1}{M(M(1-p) / p+2)}\right)} .
\end{aligned}
$$

\section{$\beta$-diversity under aggregated spatial distribution of species}

In reality, species almost always show aggregated instead of random spatial distribution due to a variety of ecological processes such as dispersal limitation, habitat filtering, and 
210 local competition. It has long been recognized that spatial aggregation of empirical species

211 can be well modeled by the negative binomial distribution (NBD, Boswell \& Patil 1970;

212 Pielou 1977). Using the same logseries SAD model as before and the NBD for aggregated

213 species spatial distribution, we can derive a prediction of $\beta_{\mathrm{P}}$ (Barton \& David 1959; He \&

214 Reed 2006; see Appendix S1 for the derivation):

$$
\begin{gathered}
\beta_{\mathrm{NBD}}=\frac{-1}{\ln (1-p)} \sum_{n=1}^{\infty} \frac{p^{n}}{n}\left(1+\frac{n}{M k}\right)^{-k} \approx \frac{\ln \left(\frac{M}{1+\lambda M}\right)+C(k, \lambda M)}{\ln (1 / \lambda)}, \\
\operatorname{Var}\left(\beta_{\mathrm{NBD}}\right)=\left[\frac{1}{S M \ln (1-p)}\left(\ln \frac{1-p e^{-\frac{1}{M}}}{1-p e^{-\frac{2}{M}}}+\frac{p e^{-\frac{2}{M}}}{M\left(1-p e^{-\frac{2}{M}}\right)}\right)\right] \\
+\left[\frac { - 1 } { S \operatorname { l n } ( 1 - p ) } \left(\sum_{n=1}^{\infty}\left(1+\frac{n}{M k}\right)^{-2 k} \frac{p^{n}}{n}\right.\right. \\
\left.\left.-\frac{-1}{\ln (1-p)}\left(\sum_{n=1}^{\infty}\left(1+\frac{n}{M k}\right)^{-k} \frac{p^{n}}{n}\right)^{2}\right)\right]
\end{gathered}
$$

where $k(>0)$ is an aggregation parameter with smaller values indicating more aggregated distribution, $C(k, \lambda M)$ is a function fully determined by $k$ and $\lambda M$ (i.e., a constant for given values of $k$ and $\lambda M$; see Appendix S1). Like equation (2), the approximation here is valid under conditions $M \gg 1$ and $\lambda \ll 1$. Note that the variance in equation (4b) includes both the variation due to species spatial distribution (the first square bracket) and the variation due to SAD (the second square bracket). This is different from the null model (2), where only the variance due to random species spatial distribution occurs.

Model (4) provides a framework to predict $\beta$-diversity from the three state variables $N, S$, and $M$ and the information about species spatial aggregation $(k)$. Different from the null $\beta$ of model (2), model (4) incorporates realistic spatial distribution of species and is expected to describe $\beta$-diversity of empirical communities (see EMPIRICAL TEST below for 

using of $\beta$-deviation.

\section{Scaling of $\beta$-deviation with sampling effort}

232 Substitute the approximate versions of equations (2) and (4a) into Kraft et al.'s

$233 \beta_{\mathrm{dev}}=\frac{\beta_{\mathrm{obs}}-\beta_{\text {null }}}{\sqrt{\operatorname{Var}_{\Pi}\left(\beta_{\text {null }}\right)}}\left(\right.$ replace $\beta_{\text {obs }}$ with $\left.\beta_{\mathrm{NBD}}\right)$, we have:

$$
\beta_{\mathrm{dev}}=\frac{C(k, \lambda M)}{\sqrt{\ln \left(\frac{2+\lambda M}{1+\lambda M}\right)-\frac{1}{2+\lambda M}}} \sqrt{\frac{S M}{\ln \left(\frac{1}{\lambda}\right)}} .
$$

Until now all the derivations in the above assume that the metacommunity is fully sampled.

That is, all the $M$ local communities (that comprise the metacommunity) are sampled. In real

shown in Bennet \& Gilbert (2016), $\beta$-deviation is subject to the effect of sampling effort.

239 Considering equation (5a), when only $m$ (out of $M$ ) local communities are sampled, the

parameter $k$ will not change but both $S(m)$ and $\lambda(m)$ will change with $m$. Nevertheless, 
$\beta_{\mathrm{dev}} \propto \sqrt{m}$

\section{EMPIRICAL TEST OF THE MODELS}

Data

We used several well-studied data sets from the literature to evaluate the analytical results

derived above. Alwyn Gentry's forest transect data (Phillips \& Miller, 2002) were used to

confirm the performance of our analytical models (2) and (4). This data set has been

previously analyzed using the $\beta$-deviation (Kraft et al., 2011; Qian et al., 2013). The data

consist of 226 plots distributed worldwide, of which 198 are 'standard' plots. Each plot is

considered as a metacommunity (Kraft et al., 2011; Qian et al., 2013) that contains ten $2 \times 50$

$\mathrm{m}$ transects (each transect is considered as a local community) where all plants with a stem

diameter $\geq 2.5 \mathrm{~cm}$ were measured and identified to species or morphospecies. Following the

previous studies, we used the 198 standard plots in this study. For each metacommunity, we

calculated the observed $\beta$-diversity $\left(\beta_{\mathrm{obs}}\right)$ and the expected $\beta$-diversity $\left(\beta_{\text {null }}\right)$, variance

$\left(\operatorname{Var}_{\Pi}\left(\beta_{\text {null }}\right)\right)$, and $\beta$-deviation $\left(\beta_{\text {dev }}\right)$ for the spatial random hypothesis (equation 2$)$. We also

computed $\beta_{\text {dev }}$ following the randomization approach of Kraft et al. (2011). We then plotted

these two $\beta_{\mathrm{dev}}$ to evaluate consistency between our analytical null model and the original

randomization approach. These two $\beta_{\text {dev }}$ were also plotted versus absolute latitude for 
that are known for each metacommunity, model (4) requires an additional aggregation

parameter $k$. We estimated it for each metacommunity by maximizing the log-likelihood

function (He, Gaston, \& Wu, 2002): $l=\sum_{i=1}^{S}\left[o_{i} \ln \left(\mathrm{E}\left(o_{i} \mid n_{i}\right)\right)+\left(M-o_{i}\right) \ln \left(1-\mathrm{E}\left(o_{i} \mid n_{i}\right)\right)\right]$

for the occupancy model $\mathrm{E}\left(o_{i} \mid n_{i}\right)=M-M\left(1+\frac{n_{i}}{M k}\right)^{-k}$, where $o_{i}$ is the number of local communities where a species with $n_{i}$ individuals is present (i.e., the occupancy). The other notations are the same as in previous sections.

To test the prediction about the power law scaling of $\beta$-deviation with sampling effort, we extracted data on $\beta$-deviation and sample size from the four different datasets reported in Bennet \& Gilbert (2016). The four datasets are: (1) plants in $6051-\mathrm{m}^{2}$ plots collected from meadow patches in the Garry Oak Ecosystem of southern British Columbia and northern Washington State; (2) plants in $1101-\mathrm{m}^{2}$ plots collected in an abandoned field at the Koffler Scientific Reserve in southern Ontario, Canada; (3) understory plants in $8550-\mathrm{m}^{2}$ forest plots collected from Mount St. Hilaire, near Montreal, Canada; and (4) diatoms in surficial sediments sampled from 492 North American lakes. More details about these datasets can be found in Bennet \& Gilbert (2016) and the references therein. The extracted $\beta$-deviation and sample size were plotted on a log-log graph and were compared against the theoretical power law of equation (5b).

\section{Test results}

The empirical tests shown in Fig. 1 confirm that our theoretical null model (equation 2)

for the random species spatial distribution accurately implements the randomization

procedure of Kraft et al. (2011). The analytical null model $\beta$-deviation has an appreciable but weaker correlation with the $\beta$-deviation computed from Kraft et al.'s randomization process 
$291\left(R^{2}>0.49\right.$, Fig. 2a). Only nine out of Gentry's 198 metacommunities show significant

292 difference between the two versions of $\beta$-deviation. Both methods are consistent in showing

293 the decreasing latitudinal gradients of $\beta$-deviation, but the gradient is appreciably somewhat

294 stronger using the analytical $\beta$-deviation (Fig. 2b, c). The Kolmogorov-Smirnov test shows

295 that the logseries describes the metacommunity SAD very well $(P>0.05)$ for 180 out of the

296198 plots (Fig. 3). All the nine "outlier" plots identified in Fig. 2 are among the few that have

297 a poor fit of logseries $(P<0.05$; Fig. 3b).

The NBD $\beta$-diversity (equation 4) performs very well in predicting empirical $\beta$-diversity

$\left(R^{2}=0.88\right.$, Fig. 4). Again, nine out of Gentry's 198 metacommunities show significant

departure from the prediction (note seven of those nine are the same "outlier" plots as in Fig.

2). The empirical relationships between $\beta$-deviation and sampling effort employed by Bennett

$\&$ Gilbert (2014) to argue against the use of $\beta$-deviation follow our theoretical power-law

scaling equation (5b) very well, as long as the sample size is not too small (i.e., $m>30$, Fig.

\section{DISCUSSION}

In this paper we have developed analytical $\beta$-diversity for random and aggregated spatial

$\beta$-diversity which would otherwise has to be implemented by randomization (Kraft et al., 
of two differences between our null model and the original randomization approach. The

second difference is that we do not preserve the abundance in each local community. Previous

Appendix S2), while the randomization approach uses the empirical SAD that could be

real applications almost all empirical SADs are based on sampling data and themselves are

not true metacommunities. This sampling problem is one of the major criticisms of Kraft et

$\beta$-diversity based on the theoretically justified logseries SAD of metacommunity provides a

desirable solution.

Unlike the randomization process, our $\beta$-diversity models do not require abundances of individual species but only the data on metacommunity size $(N)$ and total richness $(S)$ (for null $\beta$-diversity equation 2 ) or $N$ and $S$ plus spatial distribution parameter $k$ (for the NBD $\beta$-diversity equation 4). More significantly, these analytical results reveal the dependence of

$\beta$-diversity on $N, S$, species spatial pattern and sampling effort and thus offer unambiguous interpretation on $\beta$-deviation which is otherwise not obvious and has been a subject of debate randomized $\beta$-deviation. Our discussion will make it clear that $\beta$-deviation is an important and solid diversity measure for testing community assembly mechanisms and the 
much-criticized sampling effect on $\beta$-deviation (Bennett \& Gilbert, 2016) can be easily dealt with using the simple scaling law of equation (5).

The fact that the null $\beta$-diversity of equation (2) is derived from the assumption of random spatial distribution of species clearly indicates that the randomization procedure of Kraft et al. (2011) is for randomizing spatial distribution of species, not for correcting for the effect of regional $(\gamma)$ diversity on $\beta$-diversity as originally interpreted. Hence, the $\beta$-deviation is a metric measuring the effect size of non-random species spatial distribution on $\beta$-diversity for a given SAD.

The misinterpretation of $\beta$-deviation as "a standard effect size of $\beta$-diversity deviations from a null model that corrects for $\gamma$ dependence" (Kraft et al., 2011) has raised much concern about the null model and led to attempts to develop alternative null models or metrics to truly correct for " $\gamma$ dependence" (Qian et al., 2013; Ulrich et al., 2017, 2018). It is clear from equation (5a) that $\beta$-deviation for a metacommunity with a fixed number of local communities ( $M$; can also be thought as a fixed sampling design) is fully determined by a non-linear, interactive function of the two parameters $k$ and $\lambda$ for the metacommunity (Fig. S1 in Appendix S1). It is also known that the parameter $\lambda$ is a function of the ratio between total abundance $N$ and the $\gamma$-diversity $S$ (Harte, 2011). Given the well-known non-linear relationship between $N$ and $S$, it is inevitable for $\beta$-deviation to be dependent on $S$ (imagine two systems having the same $M$ and $k$ : to maintain a constant $\beta$-deviation they must have the same $\lambda$, which requires the same $N / S$ ratio, which can only be true for the same $S$ value). This further clarifies that $\beta$-deviation is not correcting for the " $\gamma$ dependence".

There are a number of alternative approaches used in the literature that are closely related with the null model investigated here, but they all suffer from similar problems. Qian et al. 
360 (2013) and Xu et al. (2015) analyzed the raw (i.e., $\left.\beta_{\text {obs }}-\beta_{\text {null }}\right)$, instead of the standardized,

$361 \beta$-deviation. From our models (2) and (4), it is clear that the raw $\beta$-deviation is also dependent

362 on $\gamma$, thus not serving as a correction for the $\gamma$-dependence. A more fundamental problem with

363 the raw $\beta$-deviation is that it is not a measure of effect size and thus is of little use for

364 comparing the effect of species aggregation on $\beta$-diversity. Ulrich et al. (2018) proposed a

365 "fixed-fixed" null model that preserves both row and column sums of the community matrix

366 to correct for the $\gamma$-dependence. However, the "fixed-fixed" null model does not have an

analytical solution and its ecological interpretation is not clear.

Another major criticism on $\beta$-deviation is that it is sampling-effort dependent, meaning 
for inferring community assembly rules. The derivation of the analytical version of the null model in this study clarifies the interpretation of the model and addresses the problem that the $\beta$-deviation is subject to the effect of sampling effort. The requirement for species abundance data by the original randomization null model can seriously limit its application in systems of large spatial extent. Data on abundance of individual species are no longer needed for the theoretical model derived here as long as the size of the metacommunity is known. This affords the use of the theoretical model to systems of large extent and can take advantage of the occurrence data available for many taxa (e.g., birds, amphibians, mammals, and plants;

Gaston et al., 2007; McKnight et al., 2007; Buckley \& Jetz, 2008; Maitner et al., 2018).

Coupling with these data, our analytical model offers a possibility to explore the global pattern and underlying drivers of $\beta$ diversity.

One potential limitation of this study is the assumption of logseries for metacommunity SAD. In situations where the true SAD does not follow logseries, our model could elevate type I error (i.e., the $\beta$-deviation tends to reject a true hypothesis that communities are randomly assembled in space). That is, deviation of the true SAD from logseries will weaken the accuracy of predictions from our models (Fig. 3). As for the power-law scaling of $\beta$-deviation with sampling effort, both our simulation (Appendix S2) and the empirical data from various sources (Fig. 5) show that it is robust to variations in SAD. The ecological interpretation of $\beta$-deviation revealed by our analytical analyses does not suffer from the SAD assumption either. Hence, among the three major implications of our analytical results, only the application in estimating $\beta$-deviation from occurrence data could potentially be affected by the assumption. Because logseries for metacommunity SAD is theoretically justified (Hubbell, 2001; Pueyo et al., 2007; Harte, 2011) and well supported in numerous empirical 
tests (White et al., 2012; Baldridge et al., 2016), our theoretical models are of strong practical

significance, as exemplified by the majority of Gentry's forest plots (Figs. 1-4). However, system is far from logseries.

\section{ACKNOWLEDGEMENTS}

We thank late Dr. Alwyn H. Gentry and his co-workers for collecting the invaluable forest

transect data set and thank the Missouri Botanical Garden for making the data publicly

available. DX thanks Prof. John Harte for generously sending a copy of his book (Harte,

\section{REFERENCES}

Baldridge, E., Harris, D. J., Xiao, X., \& White, E. P. (2016). An extensive comparison of species-abundance distribution models. PeerJ, 4, e2823. doi:10.7717/peerj.2823

Barton, D. E., \& David, F. N. (1959). Contagious occupancy. Journal of the Royal Statistical Society. Series B (Methodological), 21(1), 120-133.

Bennett, J. R., \& Gilbert, B. (2016). Contrasting beta diversity among regions: how do classical and multivariate approaches compare? Global Ecology and Biogeography, 25(3), 368-377. doi:10.1111/geb.12413

Boswell, M. T., \& Patil, G. P. (1970). Chance mechanisms generating the negative binomial distributions. In G. P. Patil (Ed.), Random counts in models and structures (pp. 3-22). University Park, Pennsylvania: Pennsylvania State University Press. 
Buckley, L. B., \& Jetz, W. (2008). Linking global turnover of species and environments. Proceedings of the National Academy of Sciences, 105(46), 17836-17841. doi:10.1073/pnas.0803524105

Chao, A., \& Chiu, C.-H. (2016). Bridging the variance and diversity decomposition approaches to beta diversity via similarity and differentiation measures. Methods in Ecology and Evolution, 7(8), 919-928. doi:10.1111/2041-210X.12551

Crist, T. O., Veech, J. A., Gering, J. C., \& Summerville, K. S. (2003). Partitioning species diversity across landscapes and regions: a hierarchical analysis of $\alpha, \beta$, and $\gamma$ diversity. The American Naturalist, 162(6), 734-743. doi:10.1086/378901

De Cáceres, M., Legendre, P., Valencia, R., Cao, M., Chang, L.-W., Chuyong, G., ... He, F. (2012). The variation of tree beta diversity across a global network of forest plots. Global Ecology and Biogeography, 21(12), 1191-1202. doi:10.1111/j.1466-8238.2012.00770.x

Gaston, K. J., Davies, R. G., Orme, C. D. L., Olson, V. A., Thomas, G. H., Ding, T.-S., ... Blackburn, T. M. (2007). Spatial turnover in the global avifauna. Proceedings of the Royal Society B: Biological Sciences, 274(1618), 1567-1574. doi:10.1098/rspb.2007.0236

Harte, J. (2011). Maximum Entropy and Ecology: A Theory of Abundance, Distribution, and Energetics. Oxford: Oxford University Press.

He, F., Gaston, K., \& Wu, J. (2002). On species occupancy-abundance models. Écoscience, 9(1), 119-126. doi:10.1080/11956860.2002.11682698

He, F., \& Legendre, P. (2002). Species diversity patterns derived from species-area models. Ecology, 83(5), 1185-1198. 
He, F., \& Reed, W. (2006). Downscaling abundance from the distribution of species: occupancy theory and applications. In J. Wu, K. B. Jones, H. Li, \& O. L. Loucks (Eds.), Scaling and uncertainty analysis in ecology (pp. 89-108). Dordrecht: Springer Netherlands.doi:10.1007/1-4020-4663-4_5

Hubbell, S. P. (2001). The unified neutral theory of biodiversity and biogeography. Princeton, NJ: Princeton University Press.

Kraft, N. J. B., Comita, L. S., Chase, J. M., Sanders, N. J., Swenson, N. G., Crist, T. O., ... Myers, J. A. (2011). Disentangling the drivers of $\beta$ diversity along latitudinal and elevational gradients. Science, 333(6050), 1755-1758. doi:10.1126/science.1208584

Kreft, H., \& Jetz, W. (2010). A framework for delineating biogeographical regions based on species distributions. Journal of Biogeography, 37(11), 2029-2053. doi:10.1111/j.1365-2699.2010.02375.x

Maitner, B. S., Boyle, B., Casler, N., Condit, R., Donoghue, J., Durán, S. M., ... Enquist, B. J. (2018). The bien r package: A tool to access the Botanical Information and Ecology Network (BIEN) database. Methods in Ecology and Evolution, 9(2), 373-379. doi:10.1111/2041-210X.12861

McGill, B. J., Etienne, R. S., Gray, J. S., Alonso, D., Anderson, M. J., Benecha, H. K., ... White, E. P. (2007). Species abundance distributions: moving beyond single prediction theories to integration within an ecological framework. Ecology Letters, 10(10), 995-1015. doi:10.1111/j.1461-0248.2007.01094.x

McKnight, M. W., White, P. S., McDonald, R. I., Lamoreux, J. F., Sechrest, W., Ridgely, R. S., \& Stuart, S. N. (2007). Putting beta-diversity on the map: broad-scale congruence and 
coincidence in the extremes. PLoS Biology, 5(10), e272.

doi:10.1371/journal.pbio.0050272

Morlon, H., Chuyong, G., Condit, R., Hubbell, S., Kenfack, D., Thomas, D., ... Green, J. L. (2008). A general framework for the distance-decay of similarity in ecological communities. Ecology Letters, 11(9), 904-917. doi:10.1111/j.1461-0248.2008.01202.x

Myers, J. A., Chase, J. M., Jiménez, I., Jørgensen, P. M., Araujo-Murakami, A., Paniagua-Zambrana, N., \& Seidel, R. (2013). Beta-diversity in temperate and tropical forests reflects dissimilar mechanisms of community assembly. Ecology Letters, 16(2), 151-157. doi:10.1111/ele.12021

Phillips, O., \& Miller, J. S. (2002). Global Patterns of Plant Diversity: Alwyn H. Gentry's Forest Transect Data Set (Vol. 89). St. Louis: Missouri Botanical Garden Press.

Pielou, E. C. (1977). Mathematical ecology. New York: John Wiley \& Sons, Ltd. Retrieved from http://193.190.8.15/dpm/handle/0/511

Plotkin, J. B., \& Muller-Landau, H. C. (2002). Sampling the species composition of a landscape. Ecology, 83(12), 3344-3356. doi:10.1890/0012-9658(2002)083[3344:STSCOA]2.0.CO;2

Pueyo, S., He, F., \& Zillio, T. (2007). The maximum entropy formalism and the idiosyncratic theory of biodiversity. Ecology Letters, 10(11), 1017-1028. doi:10.1111/j.1461-0248.2007.01096.x

Qian, H., Chen, S., Mao, L., \& Ouyang, Z. (2013). Drivers of $\beta$-diversity along latitudinal gradients revisited. Global Ecology and Biogeography, 22(6), 659-670. doi:10.1111/geb.12020 
Tuomisto, H. (2010). A diversity of beta diversities: straightening up a concept gone awry.

Part 1. Defining beta diversity as a function of alpha and gamma diversity. Ecography, 33(1), 2-22. doi:10.1111/j.1600-0587.2009.05880.x

Tuomisto, H., \& Ruokolainen, K. (2012). Comment on "Disentangling the drivers of $\beta$ diversity along latitudinal and elevational gradients". Science, 335(6076), 1573-1573. doi:10.1126/science. 1216393

Ulrich, W., Baselga, A., Kusumoto, B., Shiono, T., Tuomisto, H., \& Kubota, Y. (2017). The tangled link between $\beta$ - and $\gamma$-diversity: a Narcissus effect weakens statistical inferences in null model analyses of diversity patterns. Global Ecology and Biogeography, 26(1), 1-5. doi:10.1111/geb.12527

Ulrich, W., Kubota, Y., Kusumoto, B., Baselga, A., Tuomisto, H., \& Gotelli, N. J. (2018). Species richness correlates of raw and standardized co-occurrence metrics. Global Ecology and Biogeography, 27(4), 395-399. doi:10.1111/geb.12711

Vannette, R. L., \& Fukami, T. (2017). Dispersal enhances beta diversity in nectar microbes. Ecology Letters, 20(7), 901-910. doi:10.1111/ele.12787

Volkov, I., Banavar, J. R., Hubbell, S. P., \& Maritan, A. (2003). Neutral theory and relative species abundance in ecology. Nature, 424(6952), 1035-1037. doi:10.1038/nature01883

White, E. P., Thibault, K. M., \& Xiao, X. (2012). Characterizing species abundance distributions across taxa and ecosystems using a simple maximum entropy model. Ecology, 93(8), 1772-1778. doi:10.1890/11-2177.1

Xing, D., \& He, F. (2019). Environmental filtering explains a U-shape latitudinal pattern in regional $\beta$-deviation for eastern North American trees. Ecology Letters, 22(2), 
522 Xu, W., Chen, G., Liu, C., \& Ma, K. (2015). Latitudinal differences in species abundance

523 distributions, rather than spatial aggregation, explain beta-diversity along latitudinal

524 gradients. Global Ecology and Biogeography, 24(10), 1170-1180. doi:10.1111/geb.12331

526 Zhang, C., He, F., Zhang, Z., Zhao, X., \& von Gadow, K. (2020). Latitudinal gradients and ecological drivers of $\beta$-diversity vary across spatial scales in a temperate forest region. 
530 Figure $1 \beta$-diversity of Gentry's 198 forest plots under the null model of random spatial

531 distribution of species. (a) Relationship between the expected $\beta$-diversity calculated using

532 equation (2a) and the randomization approach of Kraft et al. (2011). (b) Relationship between

533 standard deviation of $\beta_{\text {null }}$ calculated using equation (2b) and the randomization approach.

534 Sizes of the points are proportional to the values of logarithm of the number of species. The

535 dashed lines are 1:1 line. $R_{1: 1}^{2}$ describes the goodness-of-fit of the 1:1 line to the data, while

$536 \quad R_{x: y}^{2}$ is the $R^{2}$ between $x$ and $y$.

537 Figure $2 \beta$-deviation for Gentry's 198 forest plots. (a) Relationship between $\beta$-deviation

538 calculated using the analytical equation (2) and the randomization approach. Error bars

539 represent $1.96 \mathrm{SD}$ of $\beta$-deviation due to species abundance distribution (equation 3 ). The

540 dashed line is 1:1 line. $R_{1: 1}^{2}$ describes how well the 1:1 line fits the data. (b) Relationship of

$541 \beta$-deviation with latitude computed using the analytical solution, and (c) the randomization

542 approach. The solid lines in (b, c) show ordinary least squares regression. $R_{x: y}^{2}$ is the $R^{2}$

543 between $x$ and $y$. Filled points highlight the nine plots where the two $\beta$-deviations are

544 significantly different.

545 Figure 3 Goodness-of-fits of the logseries SAD to Gentry's data. (a) Histogram of $P$-value of

546 the Kolmogorov-Smirnov (KS) test for the logseries SAD model. (b) Relationship of the

547 difference between the analytical and randomization $\beta$-deviation with $P$-value of the KS test.

548 Filled points in (b) are plots where the two $\beta$-deviations are significantly different (same as in

549 Figure 2). The dashed vertical line indicates $P=0.05$.

$550 \quad$ Figure 4 Relationship between predicted $\beta$-diversity using model (4) and observed

$551 \beta$-diversity for Gentry's 198 forest plots. The grey dashed error bars represent $1.96 \mathrm{SD}$ due to

552 both species abundance distribution and species-level spatial distribution, with the black solid 
portions due to species-level spatial distribution only. The dashed line is the $1: 1$ line. Filled

554 points represent the nine plots where the observed $\beta$-diversity differ significantly from the

555 prediction. $R_{1: 1}^{2}$ describes the goodness-of-fit of the 1:1 line to the data, while $R_{x: y}^{2}$ is the $R^{2}$

556 between the two $\beta$-diversity.

$557 \quad$ Figure 5 Relationships of $\beta$-deviation with sampling effort for the four different datasets

558 from Bennett \& Gilbert (2016). The grey lines are the predictions of the derived power-law

559 with scaling exponent of 0.5 (equation $5 b$ ). The figure numbers in the legend refer to figures

560 in Bennett \& Gilbert (2016) where the data were extracted.

561 
bioRxiv preprint doi: https://doi.org/10.1101/2020.04.19.049163; this version posted April 20, 2020. The copyright holder for this preprint (which

was not certified by peer review) is the author/funder, who has granted bioRxiv a license to display the preprint in perpetuity. It is made available under aCC-BY 4.0 International license.

562

Figure 1
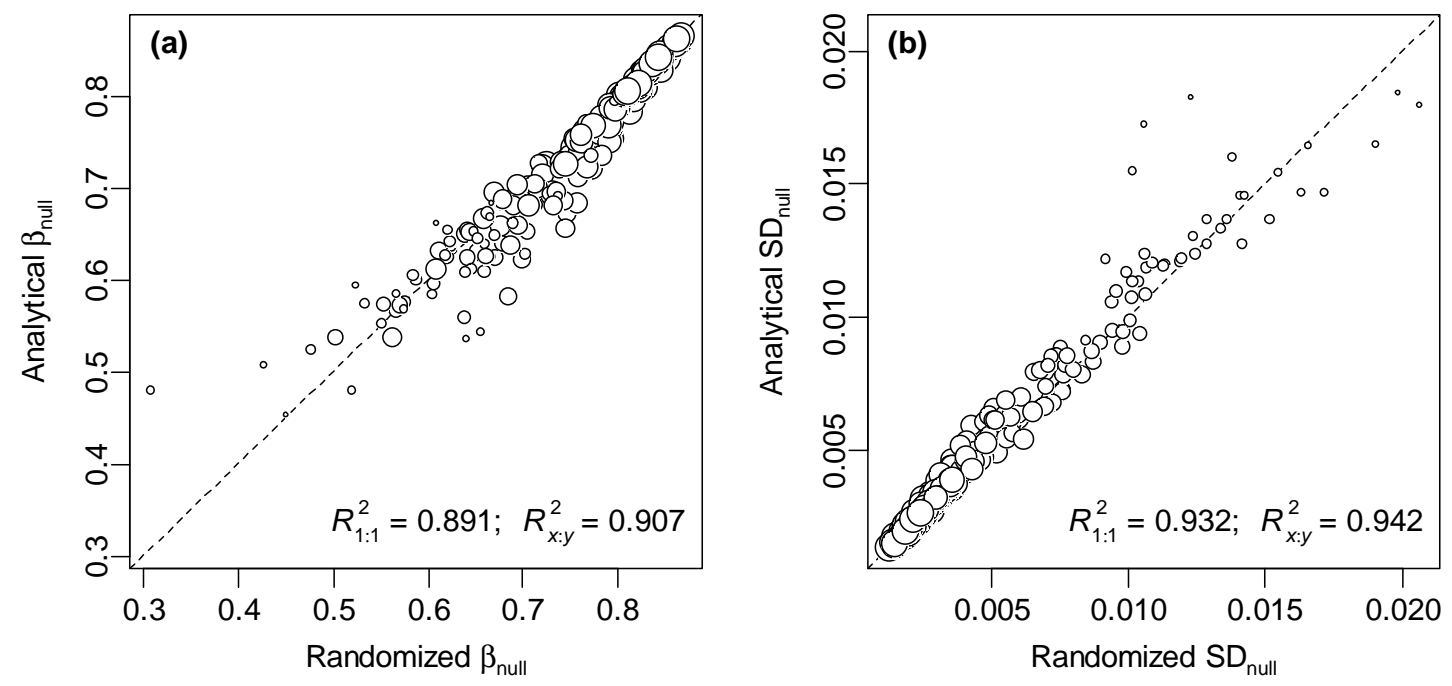

563

564 
bioRxiv preprint doi: https://doi.org/10.1101/2020.04.19.049163; this version posted April 20, 2020. The copyright holder for this preprint (which

was not certified by peer review) is the author/funder, who has granted bioRxiv a license to display the preprint in perpetuity. It is made available under aCC-BY 4.0 International license.

$565 \quad$ Figure 2
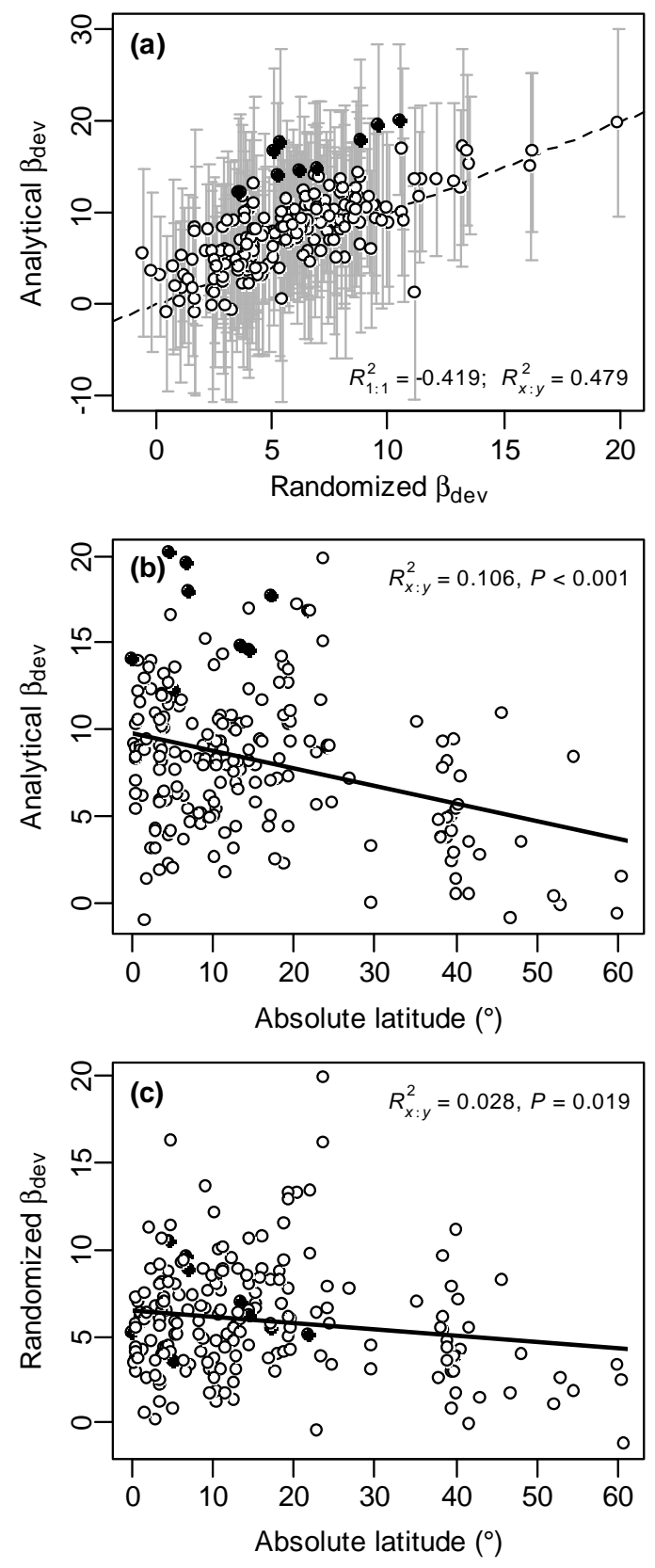

567 
bioRxiv preprint doi: https://doi.org/10.1101/2020.04.19.049163; this version posted April 20, 2020. The copyright holder for this preprint (which

was not certified by peer review) is the author/funder, who has granted bioRxiv a license to display the preprint in perpetuity. It is made available under aCC-BY 4.0 International license.

$568 \quad$ Figure 3

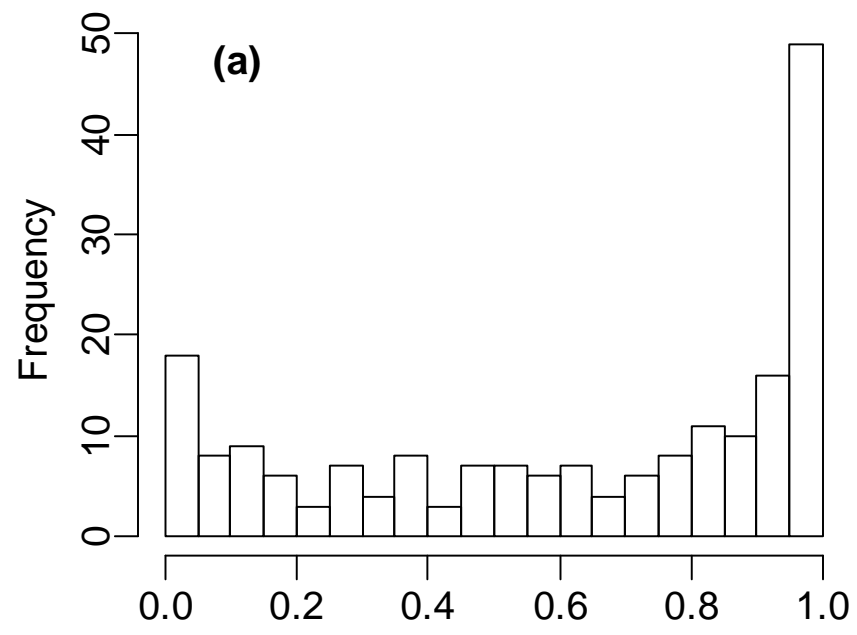

569

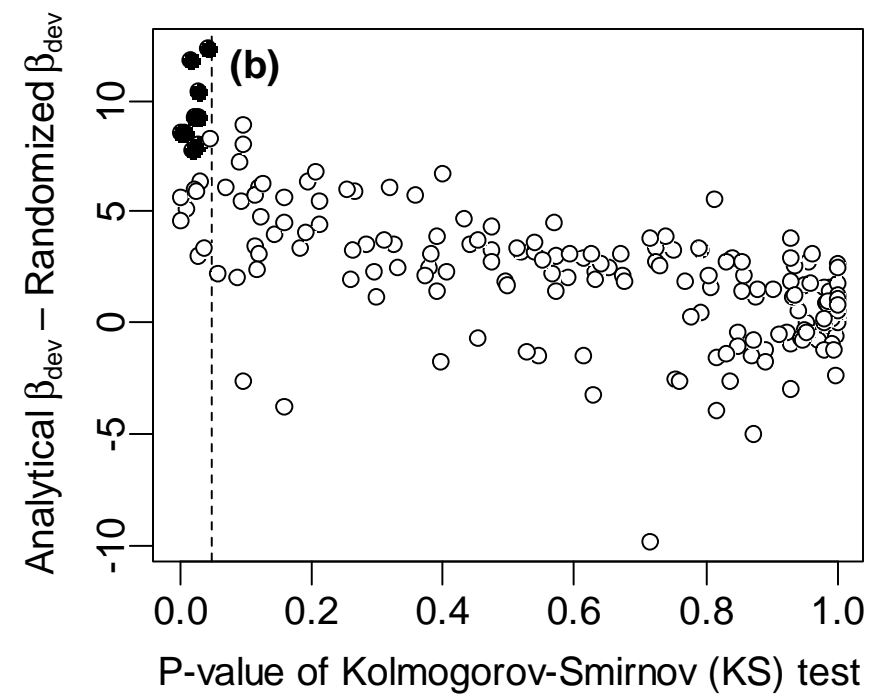

570 
bioRxiv preprint doi: https://doi.org/10.1101/2020.04.19.049163; this version posted April 20, 2020. The copyright holder for this preprint (which was not certified by peer review) is the author/funder, who has granted bioRxiv a license to display the preprint in perpetuity. It is made available under aCC-BY 4.0 International license.

\section{$571 \quad$ Figure 4}

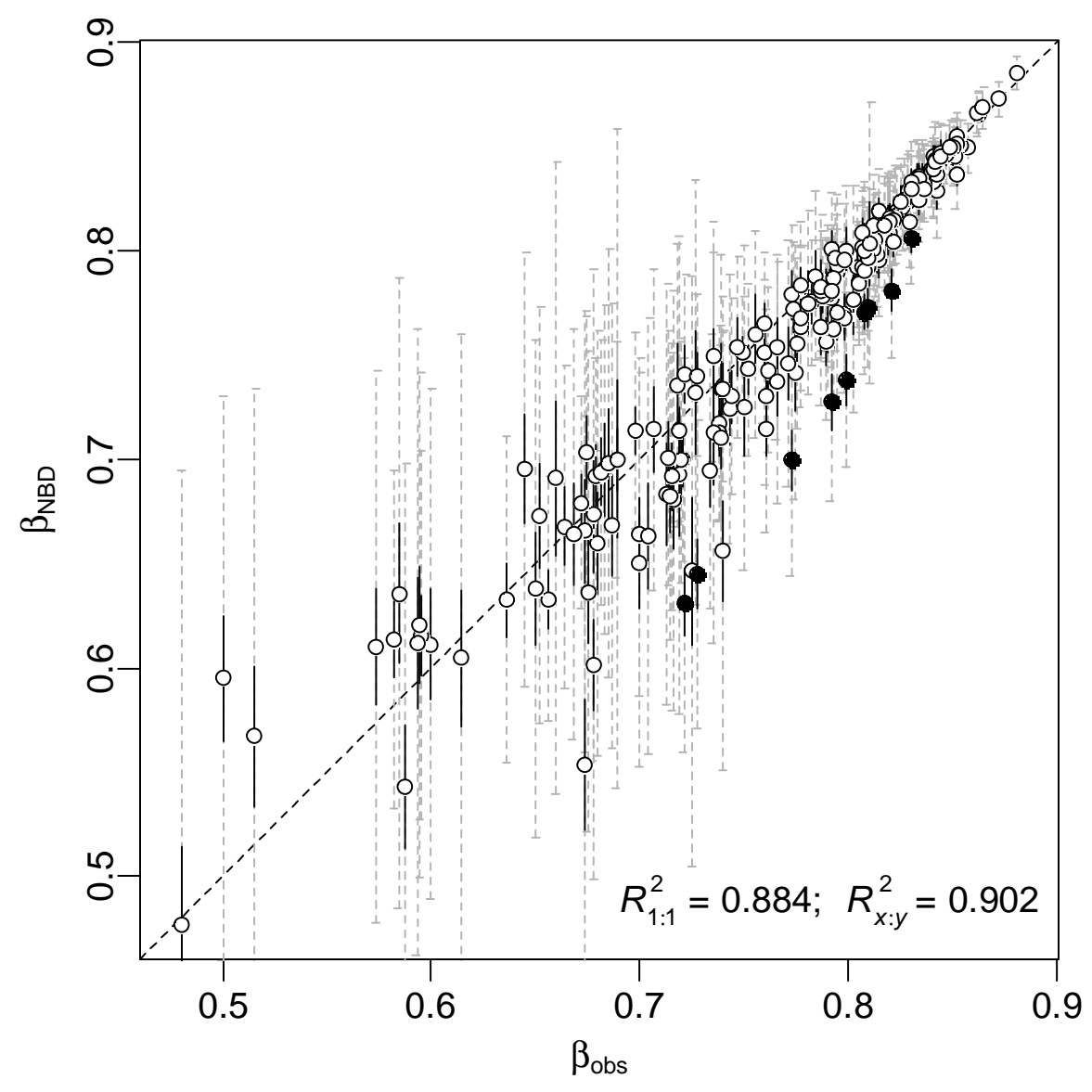

572

573 
bioRxiv preprint doi: https://doi.org/10.1101/2020.04.19.049163; this version posted April 20, 2020. The copyright holder for this preprint (which

was not certified by peer review) is the author/funder, who has granted bioRxiv a license to display the preprint in perpetuity. It is made available under aCC-BY 4.0 International license.

\section{$574 \quad$ Figure 5}

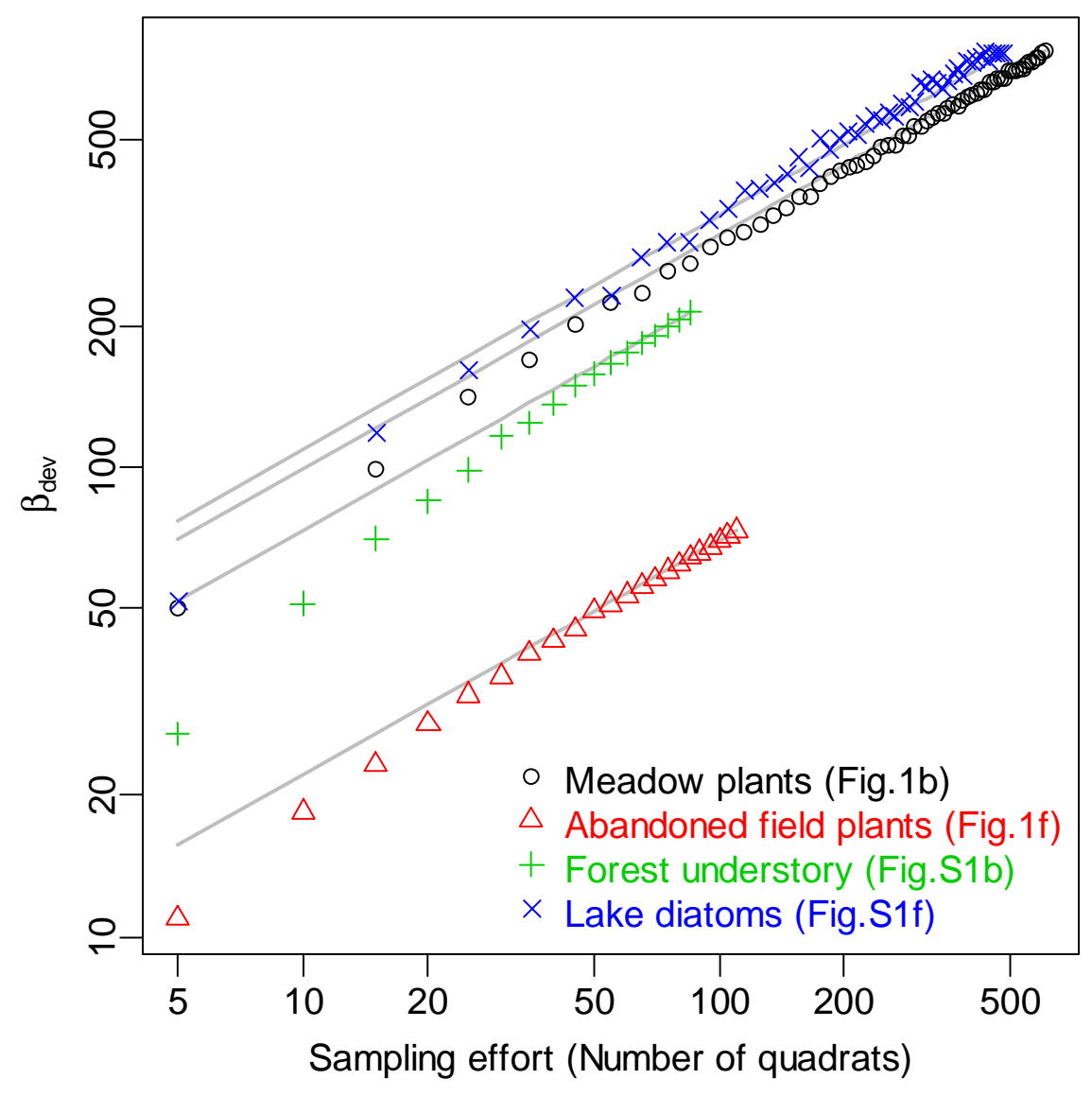

575

576 